\title{
Designing biosensor for detection of water borne pathogens
}

\section{Introduction}

The water borne pathogenic microorganisms are the biggest threat to human health. Many of such bacteria and fungi are bye products of industrial wastes, human disposals, animal excreta and hospital used products etc. Many cattle, plants and human beings get affected by the usage of contaminated water. Safe water is most essential commodity for survival of animals and plants. Water contaminated with pathogenic fungi and bacteria used for drinking, food making and cleaning purposes may lead to infections in digestive tract or may increase the population of pathogenic species in our Biome. According to WHO, around $4.1 \%$ of the diarrhea cases are accounted due to dirty and unsafe water supply. The children and immuno compromised people are more prone to such pathogens. ${ }^{1,2}$ Although a number of methods are known for detection of toxic materials in water, however, the biodiversity in water borne pathogens needs selective and sensitive detection.

It has been observed by us through the patients data collected in real time from Tezas Microbiology Laboratory, Allahabad, India that the most sacred river Ganga contains 8 pathogenic bacteria and 22 pathogenic fungal strains that have been affecting human health in this particular region. According to the above mentioned data most prevalent bacterial infections and their causal organisms are Botulism (Clostridium botulinum), Cholera (Vibrio cholerae), Escherichia coli infection, Dysentery (genera Shigella and Salmonella), Lep- tospirosis (genus Leptospira) and Typhoid fever (Salmonellatyphi). The most prevalent fungal species are Absidia, Acremonium, Mucorcircinelloides and Candida albicans. The major factors to deal with such issues of contamination is detection and prevention. Although there are number of methods e.g. q PCR, gene markers, direct observation and mass spectrometry for the detection of microorganisms but none is as handy, economic and specific as molecular beacons. The application of a biosensing methodology which can detect many pathogenic water borne fungal and bacterial strains through a single probe can make the process handy and reduce the time of detection by many folds.

The DNA based probes have proven to be specific and precise way of detecting the genomic sequences directly. In this dimension molecular beacons are very useful over the traditional methods of DNA based pathogen detection probes. The technology of molecular beacons has already been used earlier for living cell detection by DNA-RNA hybridization. ${ }^{3}$ The physiology of molecular beacons works basically on the principle of Florescence Resonance Energy Transfer (FRET) as shown in Figure $1 .{ }^{4}$ Molecular beacon can utilize a novel design of fluorescence resonance energy transfer (FRET) and is a good candidate for use as a pathogen detector interface.

Two studies ${ }^{5,6}$ have recently been published by our research group in this direction and the work has shown how molecular beacon can be utilized to fabricate biosensor for water borne pathogenic bacterial and fungal strain species detection. The in-silico based models have been presented which can detect the corresponding conserved sequence in the target species. One of the study published on pathogenic bacterial strains has presented a single beacon probe for detection of
Volume 2 Issue 6 - 2017

\author{
Krishna Misra, Sonali Misra \\ Indian Institute of Information Technology, India
}

Correspondence: Krishna Misra, Indian Institute of Information Technology, Allahabad, India; Email krishnamisra@hotmail.com

Received: February 17, 2017 | Published: June 30, 2017

one species, ${ }^{5}$ while the other study published claims to have found the conserved sequence in the 23 pathogenic fungal species and is able to detect them all in the same shot. ${ }^{6}$ Computational approaches have been employed by us to detect the conserved and oft-repeat sequence pattern in their r-RNA sequences of twenty three water borne fungal species and nine bacterial species. In the case of fungus these species were divided into three classes- Non-Dermatophytes, Yeast, Dermatophytes. The probes were designed using common and conserved sequence (from the r-RNA sequences) among all classes and also the subsequence which was common in all the classes. The common subsequence AAT CCG GCT was utilized to design loops of molecular beacon. As the procedure of denaturation and annealing would depend on the melting temperature and therefore the number of GC containing nucleotides which were calculated in linear probes and thereafter the secondary hairpin loops were designed. The acceptor and donor fluorescent dyes that come under range of visibility (emission in visible range closer to near IR) were attached to its both ends. The process of modeling has been carried out in-silico and the protocol has been represented in the Figure 2 .
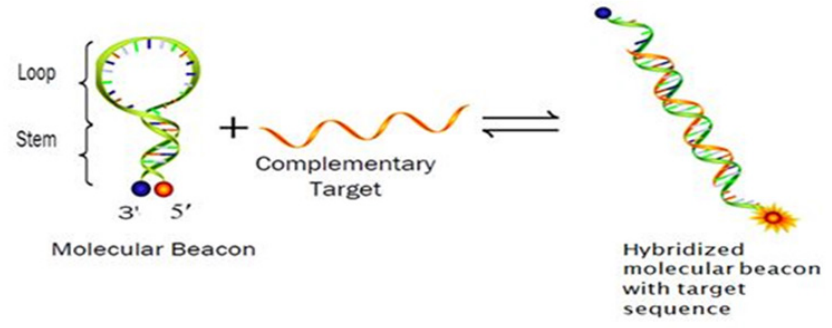

Figure I The common structure of a molecular beacon and procedure of its hybridization with the complementary sequence in the target DNA/ RNA.

In the other study [5] nine bacterial species were targeted and their conserved and common sequences were extracted from the whole genome sequence and individual molecular beacon was modeled for each specific organism. Although both the methods are important contributions in the way of effective and handy water borne pathogen detection but the approach of designing molecular beacons with specific sequences of r-RNA can prove to be a more sensitive diagnostic technique for detecting water borne fungal strains. Molecular beacons have been implemented for such proposes earlier also and in this particular case it can prove to be a great solution for collective and specific detection. The instance of few hairpin loops has been represented in Figure 3. The molecular beacon models can 
be constructed and immobilized on a solid surface so that it can be repeatedly used for the pathogen detection ${ }^{7-9}$ Figure 4.

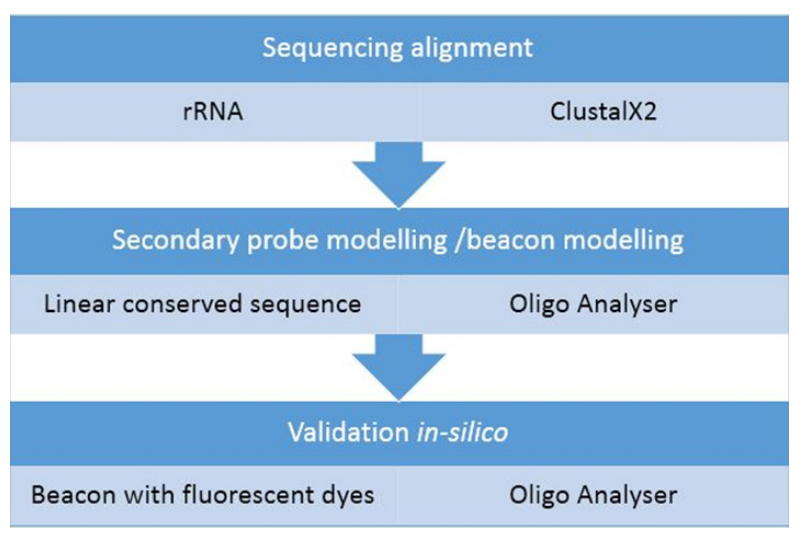

Figure 2 Flow chart describing the methodology.

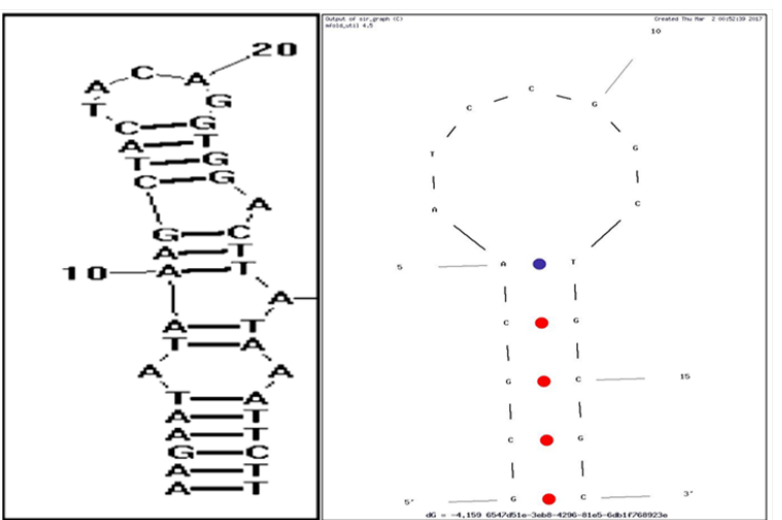

Figure 3 The molecular beacon of Campylobactor jejuni has been represented (A), molecular beacon representing the common sequence of 23 pathogenic fungal strains are shown (B).

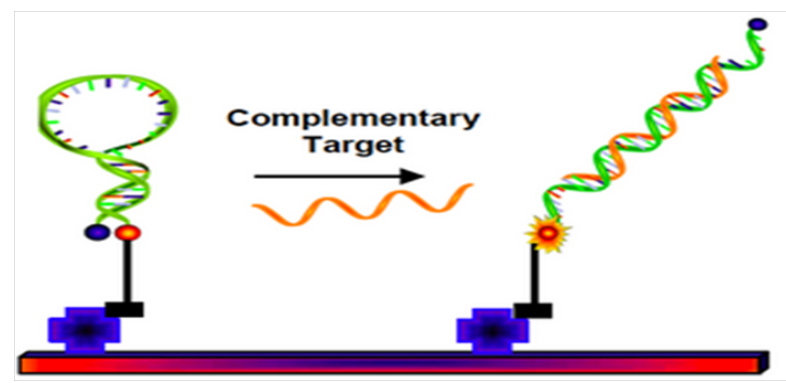

Figure 4 Biosensor model of a chip or slide on which molecular beacon is immobilized.

\section{Conclusion}

Partial gene folding method can be used to find fungal and bacterial specific naturally occurring DNA hairpins which can be identified by means of their secondary structure analysis. The designed secondary hairpin structures can be used for hybridization with probes resulting in molecular beacons.

\section{Acknowledgements}

None.

\section{Conflict of interest}

The author declares no conflict of interest.

\section{References}

1. Anaissie EJ, Stratton SL, Dignani MC, et al. Pathogenic molds (including Aspergillus species) in hospital water distribution systems: a 3-year prospective study and clinical implications for patients with hematologic malignancies. Blood. 2003;101(7):2542-2546.

2. Green BJ, Mitakakis TZ, Tovey ER. Allergen detection from 11 fungal species before and after germination. $J$ Allergy Clin Immunol. 2003;11(2):285-289.

3. Sokol DL, Zhang X, Lu P, et al. Real time detection of DNA-RNA hybridization in living cells. Proc National Academy of Science USA. 1998;95(20):11538-11543.

4. Magde D, Rojas GE, Seybold P. Solvent dependence of the fluorescence lifetimes of xanthene dyes. Photochem Photobiol. 1999;70(5):737-744.

5. Singh N, Aggarwal M, Misra K. In-Silico designing of molecular beacon probes for sensitive and rapid detection of water borne pathogenic bacteria. Proceedings of the National Academy of Sciences, India: Springer; 2015.

6. Mishra S, Misra K. Molecular beacon based biosensing for detection of pathogenic water borne multiple fungal strains: an in-silico approach. Current Bioinformatics. 2016;11:1-9.

7. Ramachandran A, Flinchbaugh J, Ayoubi P, et al. Target discrimination by surface-immobilized molecular beacons designed to detect Francisella tularensis. Biosens Bioelectron. 2004;19(7):727-736.

8. Liu X, Tan W. A Fiber-optic evanescent wave DNA biosensor based on novel molecular beacons. Anal Chem.1999;71(22):5054-5059.

9. Heyduk T, Tian. L Molecular biosensors for detecting macromolecules and other analytes; 2012. 